\title{
On Public Opinion Polls and Voters' Turnout*
}

\author{
Esteban F. Klor ${ }^{\dagger}$ and Eyal Winter
}

March 2014

* We are grateful to Oriol Carbonell-Nicolau, Eric Gould, Dan Levin, Rebecca Morton, Bradley Ruffle and Moses Shayo for very helpful discussions. We thank Hernan Meller for his valuable research assistance and Brad Coker from Mason-Dixon Polling and Research, Inc. for providing us with the data on gubernatorial elections. The paper has benefited from the comments of audiences at seminars and conferences too many to mention. All errors and mistakes in the paper remain our own.

$\dagger$ Department of Economics, The Hebrew University of Jerusalem and CEPR. Email: eklor@huji.ac.il; http://pluto.huji.ac.il/ eklor

$\ddagger$ Department of Economics and The Center for the Study of Rationality, The Hebrew University of Jerusalem. Email: mseyal@mscc.huji.ac.il 


\begin{abstract}
:
This paper studies the effects that the revelation of information on the electorate's preferences has on voters' turnout. The experimental data show that closeness in the division of preferences induces a significant increase in turnout. Moreover, for closely divided electorates (and only for these electorates) the provision of information significantly raises the participation of subjects supporting the slightly larger team relative to the smaller team. We show that the heterogeneous effect of information on the participation of subjects in different teams is driven by the subjects' (incorrect) beliefs of casting a pivotal vote. Simply put, subjects overestimate the probability of casting a pivotal vote when they belong to the team with a slight majority, and choose the strategy that maximizes their utility based on their inflated probability assessment. Empirical evidence on gubernatorial elections in the U.S. between 1990 and 2005 is consistent with our main experimental result. Namely, we observe that the difference in the actual vote tally between the party leading according to the polls and the other party is larger than the one predicted by the polls only in closely divided electorates. We provide a behavioral model that explains the main findings of our experimental and empirical analyses.
\end{abstract}

JEL Classification: C92, D72

Keywords: Experimental Study, Costly Voting, Polls. 


\section{Introduction}

In large electorates the probability of casting a pivotal vote is close to zero regardless of the actual distribution of preferences. A poll pointing to an evenly split electorate, however, may affect the voters' beliefs on the probability of casting a pivotal vote and, consequently, voters' turnout. ${ }^{1}$ Indeed, a lively debate is being carried in several countries on whether or not polls affect electoral results. A fundamental difficulty when trying to empirically assess the causal effect of public opinion polls on voters' turnout decisions is that of omitted variables. Several factors, like valence characteristics of candidates and their chosen platforms, affect not only individuals' turnout but also the public opinion polls.

This paper analyses experimentally and empirically the impact that the provision of information on the electorate's distribution of preferences has on voters' turnout. ${ }^{2}$ Our experiment compares the subjects' decision to participate in an election when they know the exact distribution of preferences of the electorate to their decisions when they only know their own preferences. Our objective is to uncover any behavioral effects that the provision of information may have on the voters. Additionally, we collected, through a survey administered at the beginning and at the end of our experiment, the subjects' estimated probabilities of casting a pivotal vote for all the different distributions of preferences. This allow us to assess whether the subjects' behavior is a consequence of their beliefs or despite thereof.

\footnotetext{
${ }^{1}$ In the U.S. presidential elections of 2004, for example, individuals that supported Ralph Nader and resided in states where the election was predicted to be close traded their votes with John Kerry's supporters that lived in states where the election was expected to be lopsided in favor of one candidate. People that traded votes felt that now their vote "really counted." As related in votepair.org/stories: "I live in Utah. The most republican state in the nation. I happen to be a democrat who voted for Gore. My vote did not count because of the stupid electoral college. By swapping my vote, I can finally have my vote count for a democrat."

${ }^{2}$ See Goeree and Großer (2005) and Taylor and Yildirim (2005) for recent theoretical studies of the effects of information on the electorate's behavior.
} 
The experimental results show that closeness in the division of preferences induces a significant increase in turnout. This is consistent with results reported in the vast related empirical literature. ${ }^{3}$ Perhaps more surprisingly, in closely divided electorates (and only for these electorates) the provision of information significantly raises the participation of subjects supporting the slightly larger team relative to the smaller team - we refer to this behavior as the bandwagon effect of polls. This behavior contradicts the qualitative predictions of the unique quasi-symmetric equilibrium of the theoretical model underlying the experiments. According to the equilibrium conditions the provision of information on the electorate's preferences should induce voters in the majority to participate less frequently because they free ride on the voting of other individuals supporting the same alternative. At the same time polls should stimulate the participation of voters in the minority to offset the advantage of the other alternative. These requirements of the mixed strategy Nash equilibrium seem counter-intuitive and are not supported by the experimental data. ${ }^{4}$

To uncover the root causes behind the bandwagon effect of polls we incorporate into the analysis the subjects' responses to the surveys. This analysis shows that the heterogeneous effect of information on subjects' turnout is driven by the subjects' (incorrect) beliefs of casting a pivotal vote. Simply put, subjects overestimate the probability of casting a pivotal vote when they belong to the team with a slight majority, and choose the strategy that maximizes their utility based on their inflated probability assessment. This conjecture was first formalized by Riker and Ordeshook (1967). To the best of our knowledge, the current paper is the first attempt to formally test this hypothesis.

\footnotetext{
${ }^{3}$ See Blais (2000), Feddersen (2004) and Merlo (2006) for recent surveys of the large existent literature on voters' turnout.

${ }^{4}$ A similar behavioral departure from mixed strategies Nash equilibrium was documented by Rapoport et al. (2002) in an experimental study of market entry with asymmetric players.
} 
The observed bandwagon effect of polls is consistent with previous experimental studies. While studying the incidence of reform in the presence of individual-specific uncertainty, Cason and Mui (2005) find that the participation rates of the majority are higher than the participation rates of the minority. Großer and Schram (2010) also conduct an experimental study of the effects of information on turnout. In particular, they examine the welfare implications of endogenous voter participation using a different experimental design that includes floating voters. They also find that the majority participates more than the minority but this difference is not statistically significant. Our experiment, like Cason and Miu's (2005), is especially designed to use the subjects as our unit of observation, granting us the possibility to differentiate between distributions with enough observations for each one to be able to perform statistically tests.

Finally, Levine and Palfrey (2005) experimentally test the predictions of Palfrey and Rosenthal (1985) whereby participation costs are heterogeneous and privately known. They find that subjects in the small team vote with higher frequency than subjects in the large team. Unlike our experimental design, theirs does not directly test for the effects of the provision of information. Perhaps more importantly, their study [as well as that of Großer and Schram (2010)] reveals the exact vote tally at the end of each round thus allowing the subjects to gain experience and learn over rounds. This was done in order to check whether the subjects' behavior converges with experience to the one predicted by the pure strategy equilibrium of the game they analyzed.

This paper's objective is not to examine the predictions of a particular model, but rather to reveal the individuals' behavioral reactions to the publication of public opinion polls. Therefore, we use a random and anonymous reassignment procedure specifically to reduce repeated game incentives and minimize the effects of learning. We believe this is the right experimental design given the objectives of our study. 
We test the external validity of our main experimental result using a newly culled data set on gubernatorial elections in the U.S. between 1990 and 2005. For these purposes we use as our proxy to closeness in the distribution of preferences the results of pre-election polls published by newspapers within one week before the elections. The empirical evidence is consistent with the experimental results. Namely, in elections where the polls pointed to a narrow margin between the two parties, the difference in the elections' vote share of the two parties is greater than the difference predicted by the polls. The effect above is not present in electoral contests that were expected to be lopsided according to the polls' predictions.

The individuals' behavioral pattern has significant implications in two different contexts. The immediate one is in the context of voting, where our results point to an interesting behavioral phenomenon that has been overlooked by the related literature. This behavior has important implications on the widespread policy debate on the desirability of publishing polls close to an election date. On the one hand, supporters of the ban claim that the observed inclination of people to vote for candidates leading in the surveys may lead to the manipulation of polls before elections by parties with vested interests. On the other hand, opponents to the measure claim that a ban on polls before elections suppresses the freedom of expression. ${ }^{5}$ Experimentally, we show that the bandwagon effect is a direct consequence of higher voter participation and not necessarily of voters changing their preferences. This suggests that a policy geared to increase voters' participation can substantially offset the effects of polls. ${ }^{6}$

The observed phenomenon has also broader implications regarding the empirical relevance of

\footnotetext{
${ }^{5}$ According to the Foundation for Information/ESOMAR a ban on the publication of opinion polls exists in 30 out of 66 countries surveyed in their study published in 2003. Nowadays, a lively debate is being conducted in several countries, like Canada, France, Ireland, The Philippines and Russia.

${ }^{6}$ Klor and Winter (2007) perform a welfare analysis of the effect of public opinion polls. We show that polls decrease the payoffs of individuals in the small team without necessarily increasing the total welfare of the population.
} 
mixed strategy equilibria in more general setups. When a player can choose between two alternatives she may use a mixed strategy only when she is indifferent between the two. In an asymmetric environment as the one proposed here, players in the small team would be indifferent between voting and abstaining only if players in the large team vote with a relatively lower frequency than players of the small team. Moreover, each player should have the correct beliefs regarding the mixed strategies used by the rest of the players. The equilibrium strategies (and beliefs) are not necessarily intuitive, especially in setups with only slight differences between the players. Thus, for these conditions to hold behaviorally probably requires that the game is played with a considerable amount of repetition to facilitate experience and learning.

We propose an alternative theoretical explanation that relaxes Nash equilibrium but is consistent with the voters' beliefs and behavior observed in the laboratory. In particular, we show that if individuals believe that in a close election the probability of voting is sufficiently high and similar for every voter regardless of team sizes, then optimal behavior with respect to these beliefs gives rise to voting patterns consistent with the ones observed in the current study. While these beliefs cannot be part of equilibrium with groups of unequal sizes, they are consistent with the documented departures from quasi-symmetric equilibrium strategies in other contexts as well.

The paper is organized as follows. The next section presents the theoretical framework underlying our experiment. A detailed description of our experimental design appears in Section 3. Section 4 shows the main experimental results of the paper. Section 5 test the external validity of our experimental results. We present an alternative theoretical explanation for the subjects' behavior in Section 6. The last section of the paper concludes. The proof of our theoretical result appears in the appendix. 


\section{Theoretical Framework}

The theoretical framework we consider is based on the seminal contribution of Palfrey and Rosenthal (1983). There are $n$ risk neutral individuals $(n \geq 3)$. Individuals have to decide between two alternatives $\{A, B\}$. The alternative is chosen via simple plurality rule; that is, the alternative with the greater number of votes is chosen. In the event of a tie each alternative is selected with equal probability. This is a collective choice problem: the chosen alternative applies to all the individuals.

Each individual has preferences over the two alternatives. Let $V$ denote the utility difference to an individual between the event that her favored alternative is elected and the event that the other alternative wins the election. ${ }^{7}$ Each individual has to decide whether to vote or abstain. ${ }^{8}$ Let us denote by $s_{i}$ the strategy of individual $i$ (let $s_{i}=1$ when individual $i$ votes and $s_{i}=0$ otherwise). All the individuals make their strategy choices simultaneously. There is a positive cost $C>0$ associated with the act of voting. $V$ and $C$ are common knowledge and identical to all the individuals. We assume that $V>2 C$.

In this setup, a rational individual votes if and only if

$$
V \times P\left(1, s_{j \neq i}\right)-C \geq V \times P\left(0, s_{j \neq i}\right),
$$

where $P$ denotes the probability that individual $i$ 's preferred alternative is chosen and $s_{j \neq i}$ is a

\footnotetext{
${ }^{7}$ Therefore, individuals are splitted regarding their preferences and have opposing preferences over the two possible outcomes. See Battaglini et al. (2010) and Houser et al. (2011) for experimental evidence on the effect that information on the state of the world has on voters' decisions.

${ }^{8}$ In the present framework voting against one's preferred alternative is strictly dominated by not voting. Therefore, we rule out this possibility and, whenever we say that an individual votes, we imply that she votes in support of her preferred alternative.
} 
profile that describes the strategy of all the individuals excluding individual $i$.

Clearly, a rational individual participates in the election only if, given the other individuals' strategies, her participation affects the probability that her preferred alternative is chosen. In other words, an individual may turn out to vote only when she is pivotal.

We analyze the game above under two different frameworks regarding the individuals' information about the distribution of preferences. The first scenario focuses on a symmetric private value model of voting. Accordingly, each voter knows the alternative that she favors and that the probability that any other individual prefers any given alternative is the same for both alternatives. The individuals' probability distributions are stochastically independent. We focus on symmetric Bayesian Nash Equilibrium (BNE) as the relevant equilibrium concept for this framework. This equilibrium concept assumes that every individual's decision to participate is independent of the alternative that she favors because of the symmetric common prior over the individuals' distribution of preferences. That is, all the individuals randomize between voting for their preferred alternative and abstaining with the same probability. (A formal definition appears in Appendix A.)

In the second scenario the number of voters favoring each alternative is commonly known. This is the framework analyzed by Palfrey and Rosenthal (1983). This complete information game has multiple Nash equilibria. The solution concept that generates unique predictions for the game is that of totally quasi-symmetric mixed strategy Nash equilibrium (QSNE). According to this equilibrium concept all the individuals supporting the same alternative use the same strategy. This strategy involves voting with a probability strictly between zero and one. Note that, unlike the BNE, in the QSNE individuals supporting different alternatives are not necessarily mixing with the same probability. (See Appendix A for a formal definition of this equilibrium concept.) 
For the purposes of our experimental study we focus on electorates of seven individuals and set $V=10$ and $C=4$. We choose an odd number of participants in each electorate to rule out equilibrium in pure strategies (except for the case where all the participants share the same preferences). In fact, there exists a unique totally mixed QSNE and a unique totally mixed symmetric BNE with electorates of seven players choosing between two alternatives. Table 1 provides the point predictions for the unique BNE and QSNE.

Note that according to the predictions of the QSNE, individuals in the minority vote with higher probability than individuals in the majority for every distribution of preferences. This result is a direct consequence of the mixed strategies equilibrium's requirement that individuals

should be indifferent between voting and abstaining. Since individuals are willing to vote only if the probability of casting a pivotal vote is positive, they have to expect that with a high enough probability the number of votes in support for each team would be equal, or differ by only one vote. To satisfy that requirement individuals supporting the large team should vote with lower probability than individuals supporting the small team.

\section{Experimental Design}

The experiment was run at the RatioLab - The Center for Rationality and Interactive Decision Theory at The Hebrew University of Jerusalem. The 84 subjects in this experiment were recruited from the pool of undergraduate and graduate students from The Hebrew University and had no previous experience in experiments related to voters' participation.

In each session 21 subjects participated as voters. The experiments were conducted via computers. Before the experiment started an experimental administrator read the instructions aloud. 
We also asked several hypothetical questions at the end of the instructions to check subjects' comprehension of the procedure (the instructions and the questionnaire are located in Appendix B). The experiment began after all subjects had solved all questions successfully. The experiment lasted for about ninety minutes. Each subject received 80 tokens as a participation fee and subsequent earnings according to the payoffs specified in the experiment. Average earnings were equal to 244 tokens. We converted each token to NIS 0.25 and paid the subjects in cash in private at the end of the session. ${ }^{9}$ Throughout the experiment we ensured anonymity and effectively isolated each subject in a cubicle to minimize any interpersonal influence that could stimulate uniformity of behavior. Communication among subjects was not allowed throughout the session.

Each experimental session entailed 20 independent rounds. In each round we randomly divided 21 subjects into three electorates of seven participants each. At the beginning of each round an equal probability rule randomly assigned each subject to one of two teams: Green or Blue. A subject earns 10 tokens if the team she prefers is selected by majority voting in an election. Voting entails a cost of 4 tokens.

The sequence of events is as follows. Subjects know that the round is divided into two stages, and that each subject will decide whether to vote or abstain in each stage. Every subject knows that her decision in one stage is independent from her decision in the other stage. In the first stage of each round each subject knows only her preferred color. She decides whether to vote or abstain. After all the participants make their decisions we proceed to the second stage of the round. In this stage subjects are told the electorate's distribution of preferences. Subjects receive no information whatsoever on the participation of subjects in the round's first stage. Subjects have to decide

\footnotetext{
${ }^{9}$ Subjects on average earned NIS 61 for roughly 90 minutes of their time. The hourly minimum wage in Israel is slightly below NIS 20. The current exchange rate is slightly above NIS 3.5 per U.S. dollar.
} 
again whether or not to vote. After all the subjects choose an action, they learn the selected teams of the first and second elections, their corresponding payoffs for the round, and their cumulative payoffs - no information is provided on the number of subjects that voted for a given team. Ties are always broken by an equal probability rule. At the end of each round subjects are randomly reshuffled between electorates and each subject's preferred color is again randomly chosen.

In addition to playing this game each subject completed a survey that asked her to assess the probability of casting a pivotal vote for each possible team size. Every subject completed the same survey twice, before the beginning of the first round and after finishing the last round.

\section{Experimental Results}

This section presents the effects of revealing information about the electorate's distribution of preferences on the subjects' turnout. To clarify the exposition we divide this section into two subsections. The first subsection presents the basic results on the impact of information provision on subjects' participation. The second subsection reports the results taking into account not only the subjects' actions but also their beliefs.

For all the tests reported below the unit of observation is the subject. For the nonparametric tests we consider, for each subject, the average across all the different rounds. This eliminates possible correlations across repeated observations of a given subject. Therefore, the statistics reported are averages of the subjects' averages. In the regression analysis, however, we use all the available data, adopting a random effects specification with the subject as the random factor. 


\subsection{The Effect of Information on Subjects' Turnout}

Figure 1 depicts the average turnout rate before the provision of information and the average turnout rate after information is revealed, as a function of the different distribution of preferences. The figure also includes the equilibrium's predicted turnout rate.

The figure indicates that a close division of preferences induces a significant increase in turnout. Whereas the average turnout rate before the provision of information is slightly below 25 percent, the average turnout rate for a distribution of teams of sizes three and four is 40 percent (the difference between the two is statistically significant with $z=3.125, p<0.001$, two-sided sign test using the normal approximation to the binomial distribution).

The provision of information for other divisions of the electorates does not have a significant impact on the subjects' turnout relative to their turnout rates before the provision of information $(p>0.8$ when the division of teams is five versus two; $p>0.65$ when the division is six versus one; and $p>0.8$ when the division is seven versus zero, all according to a two-sided sign test). Moreover, the observed rates are not substantially different from the equilibrium's prediction. The turnout rate is higher than the equilibrium's prediction for distributions of seven versus zero and five versus two, whereas turnout is lower than the equilibrium's prediction for a distribution of six versus one. For closely divided preferences, on the contrary, we observe important quantitative differences between the subjects' turnout and the predictions of the theoretical model underlying the experiment. ${ }^{10}$

\footnotetext{
${ }^{10}$ The correlation between closeness and turnout observed in the laboratory is consistent with results in the related empirical literature [see, for example, Shachar and Nalebuff (1999)]. Feddersen and Sandroni (2006) show that this correlation can be explained using a model where voters have ethical preferences. Coate and Conlin (2004) provide empirical evidence supporting the ethical voters approach. See also Degan and Merlo (2011) for an extension of the model of ethical voting to a unified approach that combines voter uncertainty and spatial heterogeneity of preferences.
} 
Although Figure 1 reveals a clear and significant effect of closeness on participation, the figure masks important and unexpected differences between teams for a given distribution of preferences. The heterogeneous effect of closeness between teams is presented in Figure 2, which decomposes turnout as a function of the size of the teams. Note that a team of size $j$ implies that the distribution of the electorate's preferences is $(j, 7-j)$.

This figure shows the most startling effect that emerges from our experiment: For closely divided electorates the effect of information on voter participation is not homogenous across teams of different sizes. In particular, the provision of information significantly raises the participation of voters supporting the slightly larger team relative to the participation of voters supporting the smaller team, thus affecting the election's results.

In other words, for closely divided electorates revealing information on subjects' preferences causes an important increase on the participation of all the subjects. Subjects that belong to teams of size three and four vote more often after learning the distribution of preferences. This effect is significantly stronger for subjects that belong to the slightly larger team. The turnout rate for subjects that belong to a team with four supporters is more than twenty percent higher than the turnout rate of subjects that belong to a team of three members. This behavior contradicts the quantitative and qualitative predictions of the theoretical model. Accordingly, members of the minority should vote with a higher probability than the members of the majority to offset the advantage of the majority. Moreover, the provision of information should induce a decrease in the turnout rate of the majority because of free riding of its members.

We do not observe a similar effect for electorates with a more lopsided division of preferences. For electorates that are not closely divided, revealing the distribution of preferences does not affect the turnout rate of subjects supporting the small team but lowers the turnout of subjects 
supporting the large team. For example, we see an important decrease in the participation of subjects after learning that they belong to a team of size seven. A similar phenomenon occurs for subjects that belong to a team of six subjects. Note that these subjects turn out in a lower frequency than that of a subject that is the sole supporter of an alternative. An analogous situation occurs when the subjects' preferences are divided between teams of five and two members. This behavior, which seems to be a consequence of free riding, is in accordance with the equilibrium's predictions.

The different effect of closeness on subjects conditional on the size of the team they support is evident from the estimation of the following participation equation:

$$
\text { Vote_Inf } f_{i, t}=1\left\{\beta_{0}+\beta_{1} \text { Vote_NoInf }_{i, t}+\beta_{2} \text { Majority }_{i, t}+\beta_{3} \text { round }_{t}+\alpha_{i} \geqq 0\right\}
$$

where $1\{\cdot\}$ is an indicator function that takes the value 1 if the left hand side of the inequality inside the brackets is greater than or equal to zero, and 0 otherwise; Vote_In $f_{i, t}$ reflects subject $i$ 's participation decision in the second stage of round $t$ after the provision of information on the distribution of preferences. The covariates account for the subject's decision in the first stage of round $t$ before the provision of information $\left(\right.$ Vote_NoIn $\left.f_{i, t}\right)$, and whether or not the subject belongs to the large team in an electoral contest $\left(\right.$ Majority $_{i, t}$ ). We also include in the analysis a time trend $\left(\right.$ round $\left._{t}\right)$ to capture the fact that subjects may systematically change their strategy as a consequence of learning from round to round; and a subject specific constant effect $\left(\alpha_{i}\right)$ that captures random disturbances (constant through time) that characterize subject $i$.

We estimate equation (4.1) separately for each different distribution of subjects' preferences 
using a random effects probit estimation. ${ }^{11}$ Table 2 presents the estimated coefficients. The table quantifies the most striking of our results: When the electorate is closely divided, the provision of information on subjects' preferences significantly raises the participation of subjects in the majority relative to the minority. The team size effect when the electorate is divided into teams of three versus four subjects is positive, large in value relative to the other coefficients and statistically significant. It increases the probability of voting by slightly over 10 percent for the average subject. We also observe a significant negative effect of rounds, pointing to a learning process that induces subjects to reduce their participation in elections. ${ }^{12}$ Interestingly, the subjects' participation in the first stage of each round does not explain their actions after the provision of information.

The subjects' behavior is qualitatively different when the difference in the number of supporters for each team is relatively large. When the difference in the number of supporters is of three or five subjects the provision of information does not affect the participation of subjects in the majority any differently than it affects the participation of subjects in the minority. For these distributions of preferences, moreover, the number of rounds elapsed does not affect participation.

Contrary to closely divided groups, when the difference in the number of supporters for each team is relatively large the best predictor for subjects' turnout after the provision of information is the subjects' actions in the first stage of each round. There are subjects that reveal a preference for participation in the first stage, and they are the ones turning out to vote in the second stage when the electorate's preferences are not closely divided. This seems to be particularly the case in lopsided contests (6 versus 1 ) where the coefficient of the first stage decision is highly statistically

\footnotetext{
${ }^{11} \mathrm{~A}$ similar estimation strategy was used in an experimental context by Frechette et al. (2003). That study's main focus is the analysis of the impact of open versus closed amendment rules in models of bargaining.

${ }^{12}$ We test the same model including the interaction between majority and number of rounds as an additional covariate. The coefficient for this covariate is not significant. This means that the significant differences between majority and minority does not disappear over rounds.
} 
significant and also large in value relative to the other coefficients. The probability of voting in the second stage is 30 percent higher for the average subject that belongs to a team of size one and voted in the first stage relative to the average subject that did not vote in the first stage. The marginal effect of voting in the first stage on the probability of voting in the second stage is 24 percent for subjects in teams of size 6 .

The fact that the provision of information significantly raises the participation of subjects in the majority relative to the minority in closely divided electorates contradicts the intuitions behind the mixed strategies Nash equilibrium concept. It also contradicts some of the intuitions behind the alternative quantal response equilibrium concept. Interestingly, however, the quantal response equilibrium predicts that the observed participation rate should be higher (lower) than that pre-

dicted by the Nash equilibrium when the participation rate predicted by the Nash equilibrium is below (above) 0.5 (Goeree and Holt, 2005). This prediction is borne out by the data both before and after the provision of information. This prediction finds additional support in the next section where we analyze the subjects' beliefs.

\subsection{The Effect of Subjects' Beliefs on Their Turnout Decisions}

This subsection incorporates into the analysis the surveys' answers to better account for the subjects' strategies. As already pointed out, these surveys, conducted at the beginning and at the end of the experiment, asked every subject to quantify the probability of casting a pivotal vote for every possible distribution of preferences. Theoretically, the equilibrium probability of casting a pivotal vote depends only on $2 C / V$, the voting cost divided by half the benefits of a victory of the subject's preferred alternative. Given that we hold both constant, the equilibrium's predicted probability of casting a pivotal vote is constant as well regardless of the distribution of preferences. 
(In our application, with a benefit of 10 tokens and a cost of 4 tokens, this probability is equal to $0.8)$.

Figure 3 depicts the average subjects' beliefs of casting a pivotal vote as a function of the size of the team. The figure includes the results of the survey taken at the beginning (labeled Survey 1 in the figure) and at the end of the experiment (Survey 2). This figure also includes the frequencies of elections in which at least one subject was pivotal based on the other subjects actual behavior.

Figure 3 shows that subjects grossly miscalculate the probability of casting a pivotal vote. Quantitatively, the subjects state a probability much lower than the theoretical and actual probabilities. Qualitatively, subjects' beliefs seem to be strongly affected by the distribution of preferences. A low probability is attributed to situations with a large difference in the number of supporters between the two teams, whereas the probability shows an important increase for closely divided teams. On average, the subjects stated a probability of $36.14 \%$ (with a standard deviation of $21.41 \%$ ) of casting a pivotal vote when the difference between the teams is one. The stated probability decreases to $25.54 \%$ and $20.48 \%$ as the differences in team sizes increases to three and five respectively (the corresponding standard deviations are $17.53 \%$ and $18.01 \%$ ). For teams of size seven the reported probability is $23.04 \%$ (the standard deviation is $27.08 \%$ ). The subjects' estimates for a close distribution of preferences is significantly different than their estimates for the rest of the distributions $(p<0.001) .{ }^{13}$

It follows from the previous subsection (see Figure 2) that not only the subjects' beliefs of casting a pivotal vote are relatively higher for closely divided electorates, but also their propensity to vote increases for these electorates. As a consequence, the actual probability of casting a pivotal

\footnotetext{
${ }^{13}$ The probability stated for distributions with a division of two versus five is significantly different than that reported for distributions of one versus six $(p<0.001)$. Neither series is significantly different from the probabilities reported for teams of size seven ( $p=0.28$ and 0.18 , respectively).
} 
vote decreases in closely divided electorates. Hence, an increase in the subjects' beliefs of casting a pivotal vote brings about a decrease in the actual probability of being pivotal.

For a given distribution of preferences, the subjects believe that they are more likely to cast a pivotal vote when they belong to the larger team. ${ }^{14}$ Contrary to our results from the previous section, the differences are statistically significant for every distribution of preferences. The $p$-value that subjects in the majority state a higher probability of casting a pivotal vote than subjects in the minority is below 0.04 for a distribution of four versus three subjects. This value decreases to 0.03 and to 0.003 as the difference between the teams increases to three and five, respectively. ${ }^{15}$

A comparison of the subjects' beliefs and their participation decisions leads us to conjecture that the subjects' behavior is at least partially accounted by their beliefs. Simply put, subjects may overestimate the likelihood of casting a pivotal vote and act rationally based on their inflated probability assessment. To test this hypothesis we estimate equation (4.1) replacing Majority M $_{i, t}$ the explanatory variable that captured the relative effect of belonging to the majority, by each subject's beliefs of casting a pivotal vote conditional on the size of the subject's team.

The estimated coefficients appear on the second panel of Table 2. The results are qualitatively similar to the ones presented on the first panel of the table; that is, subjects' beliefs explain their behavior only when the distribution of preferences is closely divided between the two teams. Intuitively, in close elections subjects believe that there is a higher probability of casting a pivotal vote when they belong to the majority. These beliefs lead subjects to increase their relative frequency of voting when they indeed belong to the large group. Quantitatively, the coefficient

\footnotetext{
${ }^{14}$ In the only exception, subjects in the second survey assigned a higher probability of casting a pivotal vote to a team of size one $(25.02 \%)$ than to a team of size six $(21.48 \%)$.

${ }^{15}$ All the conclusions above are reached using each subject's average of both surveys for a given team size. Note that the subjects' answers to the surveys are not significantly different for any team size, and our results do not change if we use either survey instead of the subjects' average of the two surveys.
} 
for the subjects' beliefs is smaller than the coefficient estimated in panel (1). In particular, a ten percentage point increase in the belief of casting a pivotal vote when belonging to a team of size four causes a five percent increase in the probability of voting of the average subject.

When the sizes of the teams are not closely divided the subjects' beliefs do not play a significant role in their decision to participate. In these situations, as was concluded before, the best predictor for a subject's participation in the second stage of a round is the subject's action in the first stage of the round.

The next section tests the external validity of our main experimental observation using data from gubernatorial elections in the U.S.

\section{Evidence from Gubernatorial Elections in the US}

This section's exercise is mainly intended to assess the external validity of our main experimental result. Using a newly culled data set on gubernatorial elections in the U.S. we test whether, in close elections, the difference in the actual vote tally between the party slightly leading according to the polls and the other party is larger than the one predicted by the polls. This hypothesis emanates directly from our experimental results and, to the best of our knowledge, has never been addressed in the vast extant empirical literature on voter turnout.

For the purposes of our empirical exercise we use as our proxy for closeness in the distribution of preferences the results of pre-election polls on gubernatorial races in the U.S., between 1990 and 2005. These polls, conducted by an independent polling firm (Mason-Dixon Polling and Research, Inc.), were published by newspaper media within one week before the elections. ${ }^{16}$ The polls are

\footnotetext{
${ }^{16}$ According to Matsusaka and Palda (1993, p. 861) "the ideal measure would be survey predictions from opinion polls taken the day before the election." Our data come very close to that ideal.
} 
supposed to be extremely accurate. They are published right before the elections and report results based only on likely voters. Therefore, the polls already incorporate other factors that affect participation (e.g. candidates' spending and mobilizations' effects). Hence, any systematic difference between the polls and the electoral results may be attributed, at least partially, to effects that the poll has on the electorate - effects that were not taken into account by the polling company.

Our data set consists of 143 gubernatorial elections in 47 states. These are all the elections between 1990 and 2005 where Mason-Dixon Polling and Research, Inc. conducted a public opinion poll within one week before the elections and where a third party did not receive more than 30 percent of the election's votes.

The main two variables of interest are the differences in the vote share for the leading party minus the vote share for the trailing party according to the polls, and the electoral results. Let us denote by

$$
D P=L_{p}-T_{p}
$$

the difference in the vote share of the leading and trailing parties according to the polls, and denote by

$$
D E=L_{e}-T_{e}
$$

the corresponding difference between the two parties according to the electoral results. ${ }^{17}$ Our exercise focuses on deviations of the electoral results from the polls predictions, $D E-D P$, and how these deviations correlate with the size of $D P$.

\footnotetext{
${ }^{17}$ For a given election the classification of the parties as leading or trailing is fixed. Therefore, $D P$ is always positive whereas $D E$ may be negative if the winner of the election is the party trailing in the published public opinion poll. This occurs for 11 observations in our sample.
} 
Table 3 presents summary statistics for the variables of interest. The table differentiates between elections where the difference in the support between the two parties according to the polls was less than 10 percentage points and the rest of the elections. ${ }^{18}$ The table illustrates first of all that the polls predictions are indeed accurate. The first column shows that the average difference predicted by the polls between the two parties is 14.8 percentage points whereas the average difference according to the electoral results is 15.3 percentage points. That is, the average error of the polls is 0.5 percentage points. The second and third columns highlight the main difference between closely divided electorates and the rest. On average, $D E-D P$ is positive for elections expected to be close and negative for the rest of the elections. Furthermore, the absolute average value of $D E-D P$ is higher for closely divided electorates than for the rest of the electorates even though the latter set of elections has a higher standard deviation. This show that on average the polls provide a more accurate prediction of the results for lopsided divided electorates.

Figure 4 depicts $D E-D P$ on the vertical axis and $D P$ on the horizontal axis. The figure includes the resulting curve according to the predicted value of $D E-D P$ based on the estimation of a fractional polynomial of $D P$, along with the confidence interval of the mean (calculated using robust standard errors). The scatter plot exhibits a pattern consistent with our experimental results. Accordingly, for polls pointing to a narrow margin between the candidates we observe a bandwagon effect, whereby supporters of the leading candidate increase their participation relative to supporters of the trailing candidate. ${ }^{19}$ This is the case for polls predicting a difference smaller than 22 percentage points between the two parties. The bandwagon effect is particularly strong for

\footnotetext{
${ }^{18}$ The chosen cutoff of 10 percent is the level of closeness that emerges endogenously from the analysis below.

${ }^{19}$ One may think that the publication of the poll may not only affect voters' turnout but their preferences as well. Since we restrict our attention to polls published within one week of the actual elections we believe that this effect is not of an important magnitude.
} 
values of $D P$ between 4 and 10 percentage points. In this range $D E-D P$ is statistically greater than zero at the $2.5 \%$ significance level. ${ }^{20}$

In electoral contests that are expected to be one-sided the above effect is not present. For these contests the predicted value of $D E-D P$ is decreasing as the difference in the support for the two parties according to the polls increases. As predicted by the theoretical models, free riding of supporters of the large party seems to be behind the negative slope of $D E-D P$ in lopsided divided electorates.

We obtain similar conclusions from the estimation of a random-effects regression model that controls for additional covariates that may affect $D E-D P$. The main covariate of interest is a dummy variable, dubbed Close, for elections where $D P<0.1 .^{21}$ In addition, the regression below includes three dummy variables. The first one, Pres, accounts for gubernatorial elections held concurrently with presidential elections. This variable help us control for possible effects that presidential elections may have on voters' preferences and turnout. The second dummy variable, Inc, is for incumbent governors running for reelection, and the third one for elections where the leading candidate according to the polls belonged to the Democratic party $($ Dem $)$. These variables aim to control for any systematic advantage that incumbents may have in their get-out-the-vote operations, or any systematic difference in these operations between Democrats and Republicans. The estimated coefficients appear below with standard errors, clustered at the state level, in

\footnotetext{
${ }^{20}$ If we restrict the estimation to be linear, $D E-D P$ is statistically greater than zero at the $2.5 \%$ significance level for every value of $D P$ lower than 0.1 .

${ }^{21}$ We choose to define an election as close when $D P<0.1$ based on the results of Figure 4. Our results do not change qualitatively or quantitatively when we use different definitions of close elections.
} 
parentheses.

$$
\begin{aligned}
(D E-D P)_{i, t}= & \underset{(0.0115)}{-0.0118}+\underset{(0.0130)}{0.0270}(\text { Close })_{i, t}+\underset{(0.0184)}{0.0337}(\text { Pres })_{t}+\underset{(0.0095)}{0.0055}(\text { Inc })_{i, t} \\
& -\underset{(0.0114)}{0.0142}(\text { Dem })_{i, t} ; R^{2}=0.1266, N=143
\end{aligned}
$$

The results indicate that the polls systematically underestimated the electoral advantage of the leading candidate only in elections expected to be close. On the contrary, the difference between the electoral outcomes and the polls' predictions is not statistically different from zero in the rest of the elections.

Summing up, the evidence presented above is consistent with our experimental results. An important caveat is in order. We need to stress that the empirical analysis is correlational in nature. This analysis cannot, by itself, establish causality or inform us about the magnitude of the bandwagon effect of polls. We present this empirical evidence simply because, when combined with the experimental results, it strengthens the case for the existence of a bandwagon effect of public opinion polls in closely divided electorates.

\section{A Behavioral Model}

Our main experimental results, confirmed using data on gubernatorial elections, cannot be accounted by the traditional rational choice approach to turnout. The results therefore call for an alternative behavioral explanation.

An alternative specification of the voters' utility function may help explain part of the behavior observed in the laboratory. According to the traditional approach each voter's benefit and cost of 
participation are not affected by whether the voter is in the losing or winning side of the contest. Several papers, mainly interested in models of sequential voting, modify the voters' utility function to take into account the fact that voters experience a desire to vote for the winner (on top of the costs and benefits explicit in rational choice models). ${ }^{22}$ This approach, while able to explain bandwagons, assumes that the very behavior we need to explain is good for the voters. Additionally, the approach does not account for the stark difference observed in the voters' behavior between elections expected to be close and the rest of the elections.

This section proposes an alternative approach that relaxes Nash equilibrium but is consistent with the voters' beliefs and behavior observed in the laboratory. The Nash equilibrium concept requires that players optimize with respect to beliefs which are consistent with the actual strategies of players. As already pointed out, no such combination can support the behavior we observe in our findings. Our experimental results may be consistent with a weaker notion of rationality. Are there "reasonable" beliefs that we can attribute to voters under which voters' best responses will be akin to the observed behavior (without these beliefs being consistent with the actual voters' strategies)?

Herein we present a set of reasonable beliefs which satisfy these conditions. If voters believe that in a close election the probability of voting is similar for all the individuals and those probabilities are sufficiently high, then optimal behavior with respect to these beliefs gives rise to voting patterns consistent with the ones documented in the previous sections. Formally,

Proposition 1: Suppose individuals believe that voters in the majority vote with probability

\footnotetext{
${ }^{22}$ Borgers (2004) mentions this possibility in a simultaneous voting game similar to the one we analyze here. Callander (2004) models this possibility explicitly in a sequential voting game by adding a positive parameter to a voter's utility function when the voter supports the winning alternative. See Morton and Williams (1999) and Battaglini et al. (2005) for experimental studies of sequential voting games.
} 
$q$ and voters in the minority vote with probability $r$, with $|q-r|<\varepsilon$, for some sufficiently small $\varepsilon>0$. If $r \geq 1 / 2$ the probability of casting a pivotal vote is higher for a voter in the majority than for a voter in the minority.

To illustrate the intuition behind the proposition let us consider the case of a closely divided electorate when individuals believe that $r$ is close to one. In this case, an individual that supports the large team believes it is very likely that her vote may break a tie. On the contrary, an individual that supports the small team believes that her team will loose the election regardless of her choice. Thus, under the conditions of Proposition 1 individuals believe that there is a higher probability of casting a pivotal vote when they support the large team. This result is consistent with the beliefs stated by the subjects in their answers to the surveys. ${ }^{23}$

The main condition behind Proposition 1 is the individuals' beliefs that all voters mix with similar probabilities. Although these beliefs cannot be part of equilibrium with groups of unequal sizes, they seem reasonable when the preferences of the electorate are almost equally split between the two alternatives. This may explain why the bandwagon effect of polls occurs only when the electorate is closely divided.

\section{Conclusions}

This paper studies the effect that information on the voters' distribution of preferences has on turnout. The main finding is that the observed increase in turnout when the distribution of preferences is closely divided is heterogenous across teams of different sizes. In particular, the increase in turnout is significantly larger for the alternative with a slight majority according to

\footnotetext{
${ }^{23}$ The subjects' beliefs are similar to a "level-1" individual best responding to "level-0" individuals in the theoretical framework developed by Crawford and Iriberri (2007).
} 
the poll. That is, polls cause a bandwagon effect whereby the frontrunner alternative increases its relative support in the elections. This effect, observed only in close elections, is not a consequence of voters changing their preferences. Rather, it is entirely driven by individuals that already supported the leading team voting with a relatively higher frequency.

We showed that the bandwagon effect of polls in closely divided electorates is a direct consequence of the subjects' beliefs. That is, for closely divided electorates we observe that subjects overestimate the probability of casting a pivotal vote and behave according to those beliefs. On the contrary, subjects' beliefs do not explain their actions in electorates that are lopsided divided. Rather, only subjects that voted with high frequencies regardless of their beliefs or the distribution of preferences are the ones that participate in lopsided elections. This paper documented the bandwagon effect not only in the laboratory but also using data from U.S. gubernatorial elections in the last fifteen years.

It is noteworthy that the bandwagon effect cannot be accounted by the intuitions derived from theoretical models that study the effect of public opinion polls on turnout. Those intuitions are based on rational individuals holding the correct beliefs for every distribution of preferences. Hereby we proposed an alternative explanation consistent with the voters' beliefs and behavior observed in the laboratory. In particular, we presented a set of reasonable beliefs that can be attributed to voters under which utility maximization yields a behavioral pattern consistent with the bandwagon effect of polls in closely divided electorates.

Summing up, this paper discovered an anomalous behavioral pattern in the laboratory; it corroborated the external validity of this behavior for large electorates; and it presented an alternative rationale for the prevalence of bandwagon effects in close elections. Clearly, much work remains to be done for us to be able to understand what causes this effect. Currently, we are exploring 
the prevalence of this effect in general environments. It seems that subjects do not fully take into account information on an ex-post asymmetric distribution in environments that are ex-ante symmetric. This conjecture, if validated in the laboratory, has implications far beyond the context of voters' turnout.

\section{References}

[1] Battaglini, Marco, Rebecca B. Morton and Thomas R. Palfrey. 2007. "Efficiency, Equity, and Timing of Voting Mechanisms." American Political Science Review 101 (3): 409-424.

[2] Battaglini, Marco, Rebecca B. Morton and Thomas R. Palfrey. 2010. "The Swing Voter's Curse in the Laboratory ." Review of Economic Studies 77 (1): 61-89.

[3] Blais, André. 2000. To Vote or not to Vote? The Merits and Limits of Rational Choice. Pittsburgh: University of Pittsburgh Press.

[4] Börgers, Tilman. 2004. "Costly Voting." American Economic Review 94 (1): 57-66.

[5] Callander, Steven. 2007. "Bandwagons and Momentum in Sequential Voting." Review of Economic Studies 74 (3): 653-684.

[6] Cason, Timothy N. and Vai-Lam Mui. 2005. "Uncertainty and Resistance to Reform in Laboratory Participation Games." European Journal of Political Economy 21 (3): 708-737.

[7] Coate, Stephen and Michael Conlin. 2004. "A Group Rule-Utilitarian Approach to Voter Turnout: Theory and Evidence." American Economic Review 94 (5): 1476-1504. 
[8] Crawford, Vincent P. and Nagore Iriberri. 2007. "Fatal Attraction: Focality, Naivete, and Sophistication in Experimental "Hide-and-Seek" Games." American Economic Review 97 (5): 1731-1750.

[9] Degan, Arianna and Antonio Merlo. 2011. "A Structural Model of Turnout and Voting in Multiple Elections." The Journal of the European Economic Association 9 (2): 209-245.

[10] Feddersen, Timothy J. 2004. "Rational Choice Theory and the Paradox of Not Voting." Journal of Economic Perspectives 18 (1): 99-112.

[11] Feddersen, Timothy J. and Alvaro Sandroni. 2006. "A Theory of Participation in Elections." American Economic Review 96 (4): 1271-1282.

[12] Frechette, Guillaume R., John H. Kagel and Steven F. Lehrer. 2003. "Bargaining in Legislatures: An Experimental Investigation of Open versus Closed Amendment Rules." American Political Science Review 97 (2): 221-32.

[13] Goeree, Jacob K. and Charles A. Holt. 2005. "An Explanation of Anomalous Behavior in Models of Political Participation." American Political Science Review 99 (2): 201-13.

[14] Goeree, Jacob K. and Jens Großer. 2005. "Welfare Reducing Polls." Economic Theory 31 (1): $51-68$.

[15] Großer, Jens and Arthur Schram. 2010. "Public Opinion Polls, Voter Turnout, and Welfare: An Experimental Study." American Journal of Political Science 54 (3): 700-717. 
[16] Houser, Daniel, Rebecca B. Morton and Thomas Stratmann. 2011. "Turned On or Turned Out? Campaign Advertising, Information and Voting." European Journal of Political Economy 27 (4): 708-727.

[17] Klor, Esteban F. and Eyal Winter. 2007. "The Welfare Effects of Public Opinion Polls." International Journal of Game Theory 35 (3): 379-94.

[18] Levine, David K. and Thomas R. Palfrey. 2007. "The Paradox of Voter Participation? A Laboratory Study." American Political Science Review 101 (1): 143-158.

[19] Matsusaka, John D. and Filip Palda. 1993. "The Downsian Voter Meets the Ecological Fallacy." Public Choice 77 (4): 855-78.

[20] Merlo, Antonio. 2006. "Whither Political Economy? Theories, Facts and Issues." In Richard Blundell, Whitney Newey and Torsten Persson (eds.), Advances in Economics and Econometrics, Theory and Applications: Ninth World Congress of the Econometric Society, Cambridge: Cambridge University Press.

[21] Morton, Rebecca B. and Kenneth C. Williams. 1999. "Information Asymmetries and Simultaneous versus Sequential Voting." American Political Science Review 93 (1): 51-67.

[22] Palfrey, Thomas R. and Howard Rosenthal. 1983. "A Strategic Calculus of Voting." Public Choice 41 (1): 7-53.

[23] Palfrey, Thomas R. and Howard Rosenthal. 1985. "Voter Participation and Strategic Uncertainty." American Political Science Review 79 (1): 62-78. 
[24] Rapoport, Amnon, Darryl A. Seale and Eyal Winter. 2002. "Coordination and Learning Behavior in Large Groups with Asymmetric Players." Games and Economic Behavior 39 (1): $111-36$.

[25] Riker, William and Peter Ordeshook. 1968. "A Theory of the Calculus of Voting." American Political Science Review 62 (1): 25-42.

[26] Shachar, Ron, and Barry Nalebuff. 1999. "Follow the Leader: Theory and Evidence on Political Participation." American Economic Review 89 (3): 525-47.

[27] Taylor, Curtis R, and Huseyin Yildirim. 2006. "Public Information and Electoral Bias." Games and Economic Behavior 68 (1): 353-375. 


\section{APPENDix A}

\section{Definition of Totally Mixed Strategies Symmetric Bayesian Nash Equilibrium: Let}

us denote by $n_{A}$ the number of voters that prefer alternative $A$ and $n_{B}$ the number of voters that prefer alternative $B$, with $n_{A}+n_{B}=n$. A totally mixed strategies symmetric BNE in this context corresponds to a probability of voting $\gamma$ that satisfies the following two conditions:

$$
\frac{2 C}{V}=\left(\frac{1}{2}\right)^{n-1} \sum_{n_{B}=0}^{n-1}\left(\begin{array}{c}
n-1 \\
n_{B}
\end{array}\right) \Pi(\gamma \mid A)
$$

and

$$
\frac{2 C}{V}=\left(\frac{1}{2}\right)^{n-1} \sum_{n_{A}=0}^{n-1}\left(\begin{array}{c}
n-1 \\
n_{A}
\end{array}\right) \Pi(\gamma \mid B)
$$

where

$\Pi(\gamma \mid A)=\sum_{i=0}^{\min \left[n_{A}-1, n_{B}\right]}\left(\begin{array}{c}n_{A}-1 \\ i\end{array}\right)\left(\begin{array}{c}n_{B} \\ i\end{array}\right) \gamma^{2 i}(1-\gamma)^{n-1-2 i}+\sum_{i=0}^{\min \left[n_{A}-1, n_{B}-1\right]}\left(\begin{array}{c}n_{A}-1 \\ i\end{array}\right)\left(\begin{array}{c}n_{B} \\ i+1\end{array}\right) \gamma^{2 i+1}(1-\gamma)^{n-2(i+1)}$

denotes the probability that a voter that supports alternative $A$ is pivotal when the rest of the voters vote with probability $\gamma$. Similarly,

$\Pi(\gamma \mid B)=\sum_{i=0}^{\min \left[n_{A}, n_{B}-1\right]}\left(\begin{array}{c}n_{A} \\ i\end{array}\right)\left(\begin{array}{c}n_{B}-1 \\ i\end{array}\right) \gamma^{2 i}(1-\gamma)^{n-1-2 i}+\sum_{i=0}^{\min \left[n_{A}-1, n_{B}-1\right]}\left(\begin{array}{c}n_{A} \\ i+1\end{array}\right)\left(\begin{array}{c}n_{B}-1 \\ i\end{array}\right) \gamma^{2 i+1}(1-\gamma)^{n-2(i+1)}$

denotes the probability that a voter that supports alternative $B$ is pivotal when the rest of the voters vote with probability $\gamma$. Thus, conditions (A.1) and (A.2) state that a voting probability $\gamma$ is a totally mixed symmetric BNE if and only if every individual is indifferent between voting and abstaining. 


\section{Definition of Totally Quasi-Symmetric Mixed Strategies Nash Equilibrium: As}

defined by Palfrey and Rosenthal (1983, p. 27), a pair of voting strategies $\left(\lambda_{A}, \lambda_{B}\right)$ is a totally quasi-symmetric mixed strategy Nash equilibrium if and only if

$$
\begin{aligned}
\frac{2 C}{V}= & \sum_{i=0}^{\min \left[n_{A}-1, n_{B}\right]}\left(\begin{array}{c}
n_{A}-1 \\
i
\end{array}\right)\left(\begin{array}{c}
n_{B} \\
i
\end{array}\right) \lambda_{A}^{i}\left(1-\lambda_{A}\right)^{n_{A}-1-i} \lambda_{B}^{i}\left(1-\lambda_{B}\right)^{n_{B}-i}+ \\
& \sum_{i=0}^{\min \left[n_{A}-1, n_{B}-1\right]}\left(\begin{array}{c}
n_{A}-1 \\
i
\end{array}\right)\left(\begin{array}{c}
n_{B} \\
i+1
\end{array}\right) \lambda_{A}^{i}\left(1-\lambda_{A}\right)^{n_{A}-1-i} \lambda_{B}^{i+1}\left(1-\lambda_{B}\right)^{n_{B}-1-i}
\end{aligned}
$$

and

$$
\begin{aligned}
\frac{2 C}{V}= & \sum_{i=0}^{\min \left[n_{A}, n_{B}-1\right]}\left(\begin{array}{c}
n_{A} \\
i
\end{array}\right)\left(\begin{array}{c}
n_{B}-1 \\
i
\end{array}\right) \lambda_{A}^{i}\left(1-\lambda_{A}\right)^{n_{A}-i} \lambda_{B}^{i}\left(1-\lambda_{B}\right)^{n_{B}-1-i}+ \\
& \sum_{i=0}^{\min \left[n_{A}-1, n_{B}-1\right]}\left(\begin{array}{c}
n_{A}-1 \\
i+1
\end{array}\right)\left(\begin{array}{c}
n_{B}-1 \\
i
\end{array}\right) \lambda_{A}^{i+1}\left(1-\lambda_{A}\right)^{n_{A}-1-i} \lambda_{B}^{i}\left(1-\lambda_{B}\right)^{n_{B}-1-i}
\end{aligned}
$$

where the right hand side of (A.3) is simply the probability that a voter supporting alternative $A$ is pivotal when the rest of the voters supporting $A$ vote with probability $\lambda_{A}$ and all the voters supporting $B$ vote with probability $\lambda_{B}$. Similarly, the right hand side of (A.4) is the probability that a voter supporting alternative $B$ is pivotal when all the voters supporting $A$ vote with probability $\lambda_{A}$ and the rest of the voters supporting $B$ vote with probability $\lambda_{B}$. Therefore, (A.3) states a sufficient and necessary condition for $\lambda_{A}$ to be a best response to $\lambda_{B}$ and (A.4) states a sufficient and necessary condition for $\lambda_{B}$ to be a best response to $\lambda_{A}$.

Proof of Proposition 1: Let us assume that there are $n$ voters, with $n \geqslant 3$ and odd. Let us say that $n_{A}$ of the voters prefer alternative $A$ and $n_{B}$ of them prefer alternative $B$, with $n_{A}+n_{B}=n$ and $n_{A}<n_{B}$. Assume first that $r=q$. The probability that an individual that prefers alternative 
$A$ is pivotal equals

$$
\sum_{i=0}^{n_{A}-1}\left(\begin{array}{c}
n_{A}-1 \\
i
\end{array}\right)\left(\begin{array}{c}
n_{B} \\
i
\end{array}\right) q^{2 i}(1-q)^{n-1-2 i}+\sum_{i=0}^{n_{A}-1}\left(\begin{array}{c}
n_{A}-1 \\
i
\end{array}\right)\left(\begin{array}{c}
n_{B} \\
i+1
\end{array}\right) q^{2 i+1}(1-q)^{n-2(i+1)}
$$

where the first term is the probability of observing a tie and the second term is the probability that alternative $A$ loses the election by one vote. Similarly, the probability that an individual that prefers alternative $B$ is pivotal equals

$$
\sum_{i=0}^{n_{A}}\left(\begin{array}{c}
n_{A} \\
i
\end{array}\right)\left(\begin{array}{c}
n_{B}-1 \\
i
\end{array}\right) q^{2 i}(1-q)^{n-1-2 i}+\sum_{i=0}^{n_{A}-1}\left(\begin{array}{c}
n_{A} \\
i+1
\end{array}\right)\left(\begin{array}{c}
n_{B}-1 \\
i
\end{array}\right) q^{2 i+1}(1-q)^{n-2(i+1)}
$$

Therefore, rearranging terms we can express the probability that an individual supporting $B$ will break a tie minus the probability that an individual supporting $A$ will break a tie as

$$
\sum_{i=0}^{n_{A}-1}\left(\begin{array}{c}
n_{B}-1 \\
i
\end{array}\right)\left(\begin{array}{c}
n_{A}-1 \\
i
\end{array}\right) \frac{i\left(n_{B}-n_{A}\right)}{\left(n_{B}-i\right)\left(n_{A}-i\right)} q^{2 i}(1-q)^{n-1-2 i}+\left(\begin{array}{c}
n_{B}-1 \\
n_{A}
\end{array}\right) q^{2 n_{A}}(1-q)^{n-1-2 n_{A}}
$$

Similarly, subtracting from the second term of (A.5) the second term of (A.6) we obtain

$$
\sum_{i=0}^{n_{A}-1}\left(\begin{array}{c}
n_{B}-1 \\
i+1
\end{array}\right)\left(\begin{array}{c}
n_{A}-1 \\
i
\end{array}\right) \frac{\left(n_{B}-n_{A}\right)}{\left(n_{B}-1-i\right)} q^{2 i+1}(1-q)^{n-2(i+1)}
$$

Thus, the probability of casting a pivotal vote is greater when an individual supports the majority group $B$ if and only if (A.7) is greater than (A.8); this is equivalent to

$$
\sum_{i=0}^{n_{A}-1}\left(\begin{array}{c}
n_{B}-1 \\
i+1
\end{array}\right)\left(\begin{array}{c}
n_{A}-1 \\
i
\end{array}\right) \frac{\left(n_{B}-n_{A}\right)}{\left(n_{B}-1-i\right)} q^{2 i+1}(1-q)^{n-1-2(i+1)}[q-(1-q)]>0 .
$$


This inequality is satisfied if, and only if, $q>1 / 2$. The more general result for $r \neq q$ with $|q-r|<\varepsilon$, for some $\varepsilon>0$ follows immediately from the fact that the probability of casting a pivotal vote is continuous on $q$ and $r$. 
Figure 1: Average Turnout Rate by Distribution of Preferences

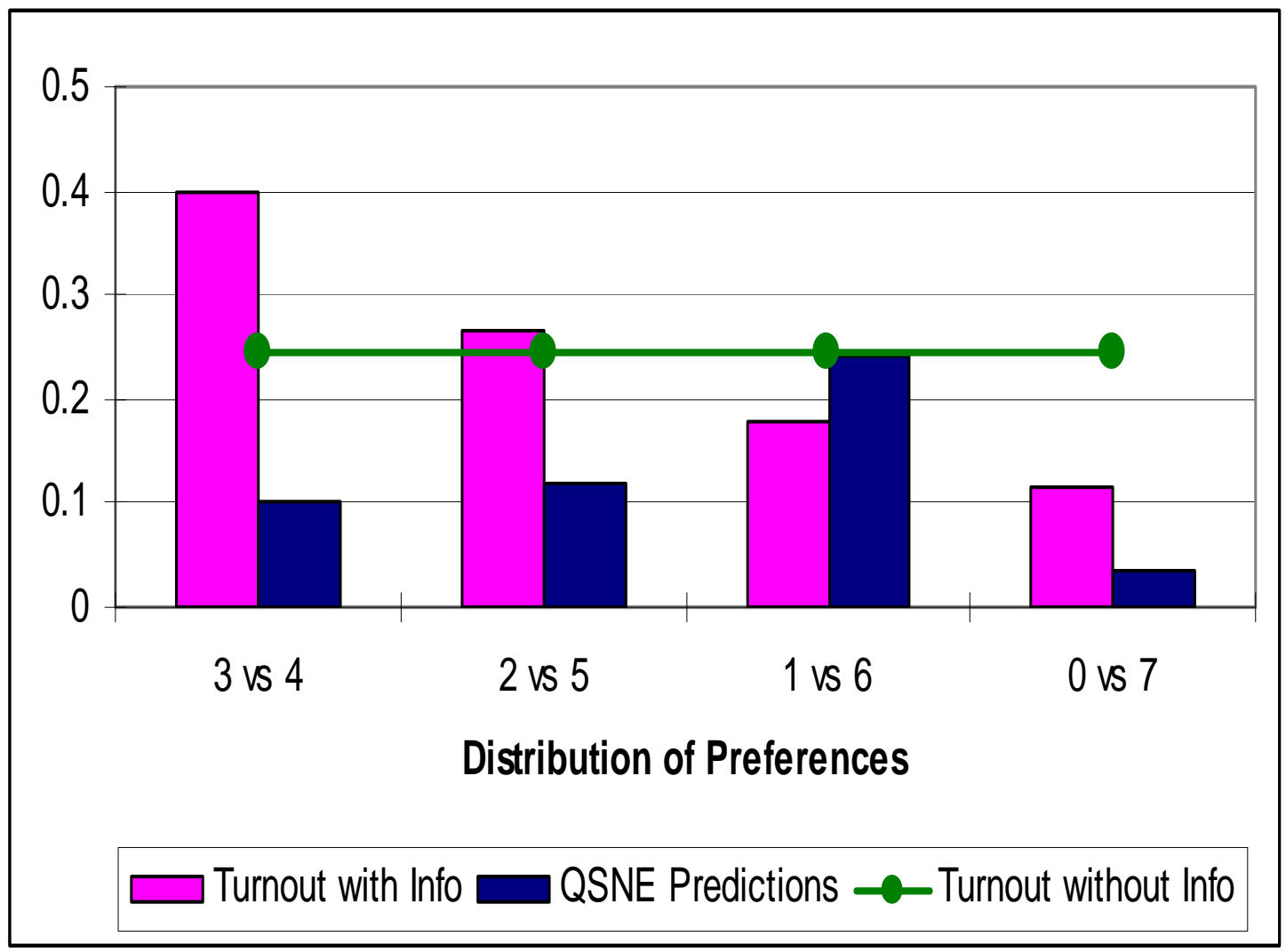


Figure 2: Average Turnout Rate by Team Size

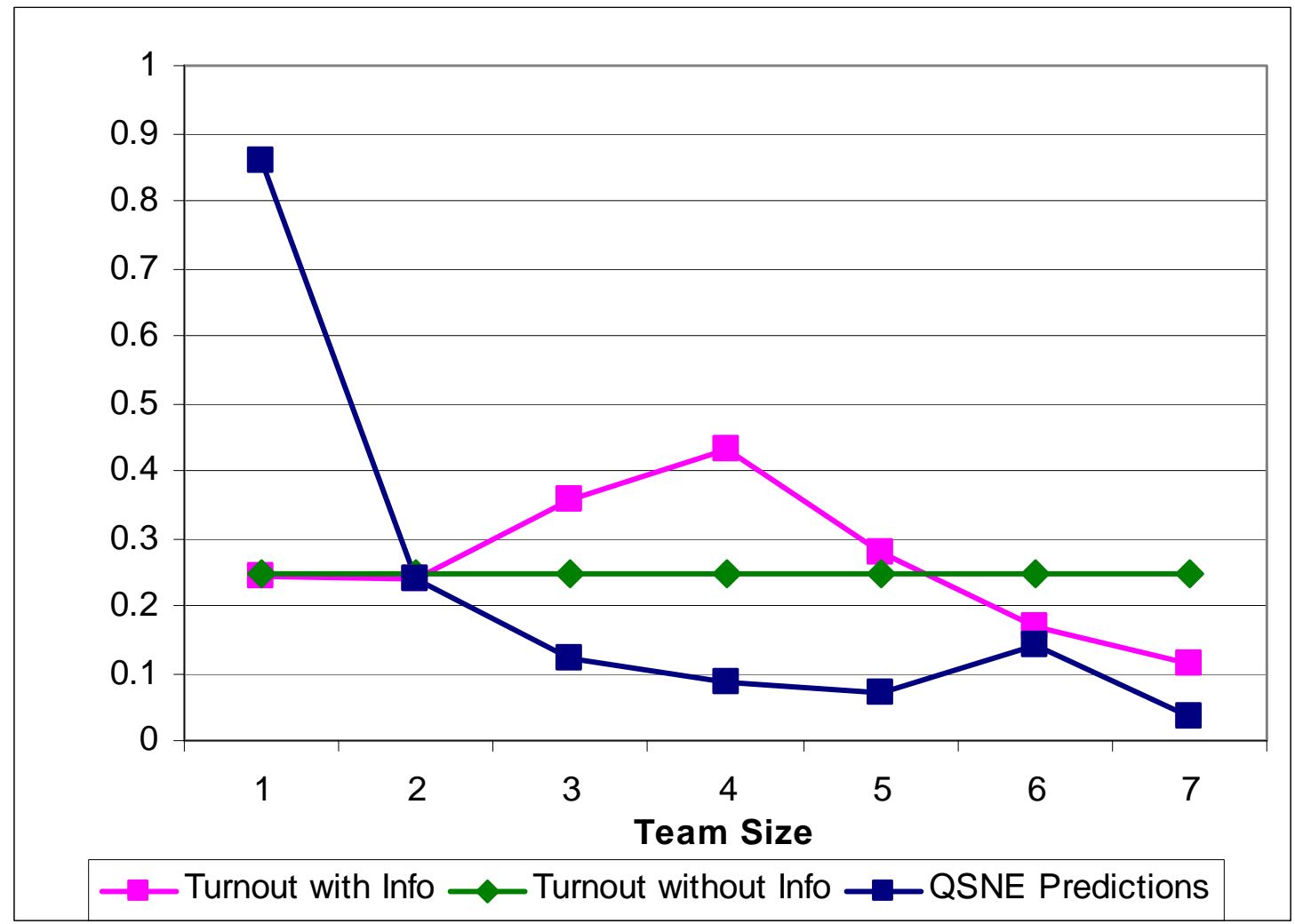


Figure 3: Subjects' Beliefs of Casting a Pivotal Vote by Team Size

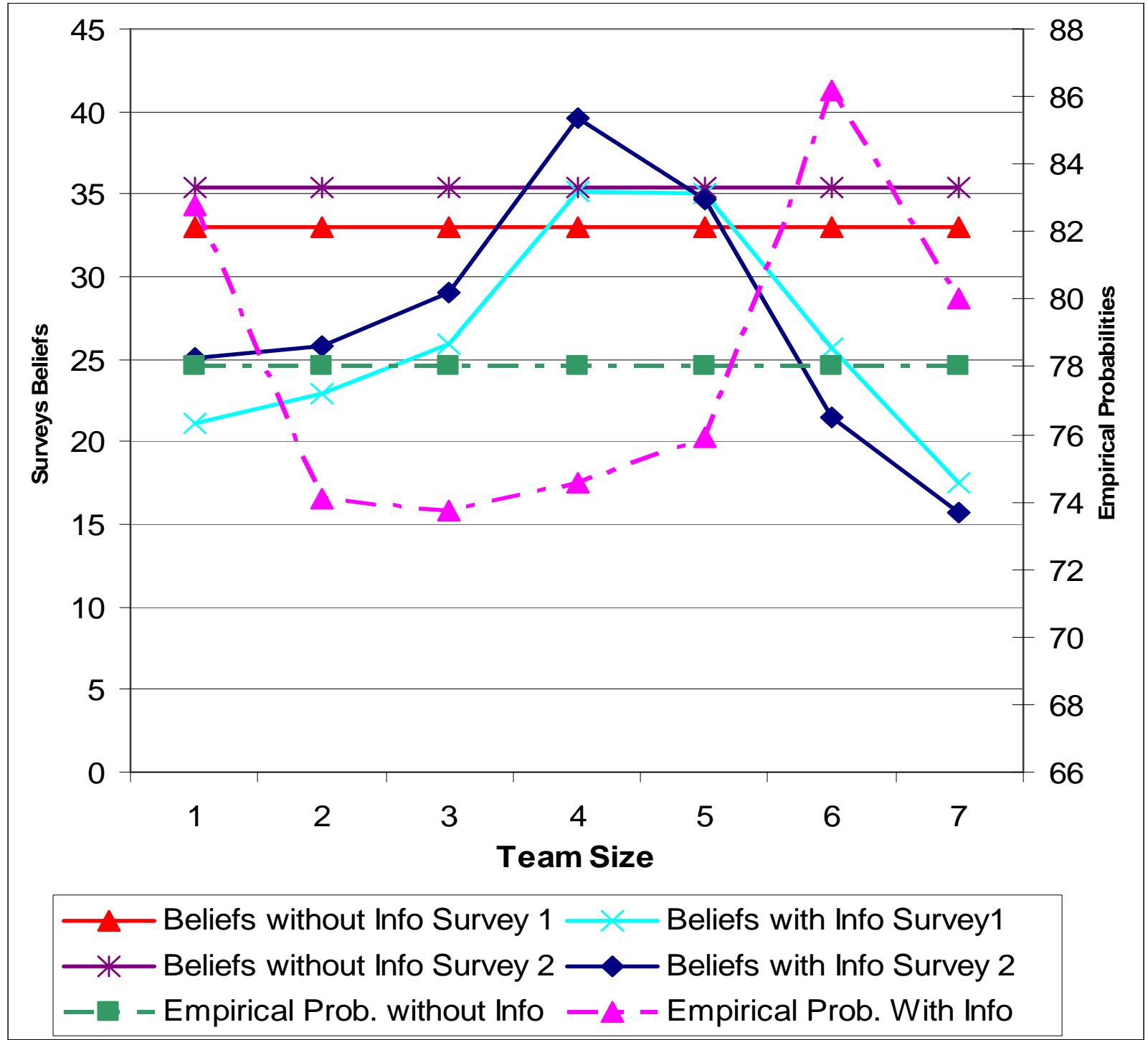


Figure 4: Deviation of Electoral Results (DE) from Polls Predictions (DP) for Gubernatorial Elections, 1990 - 2005.

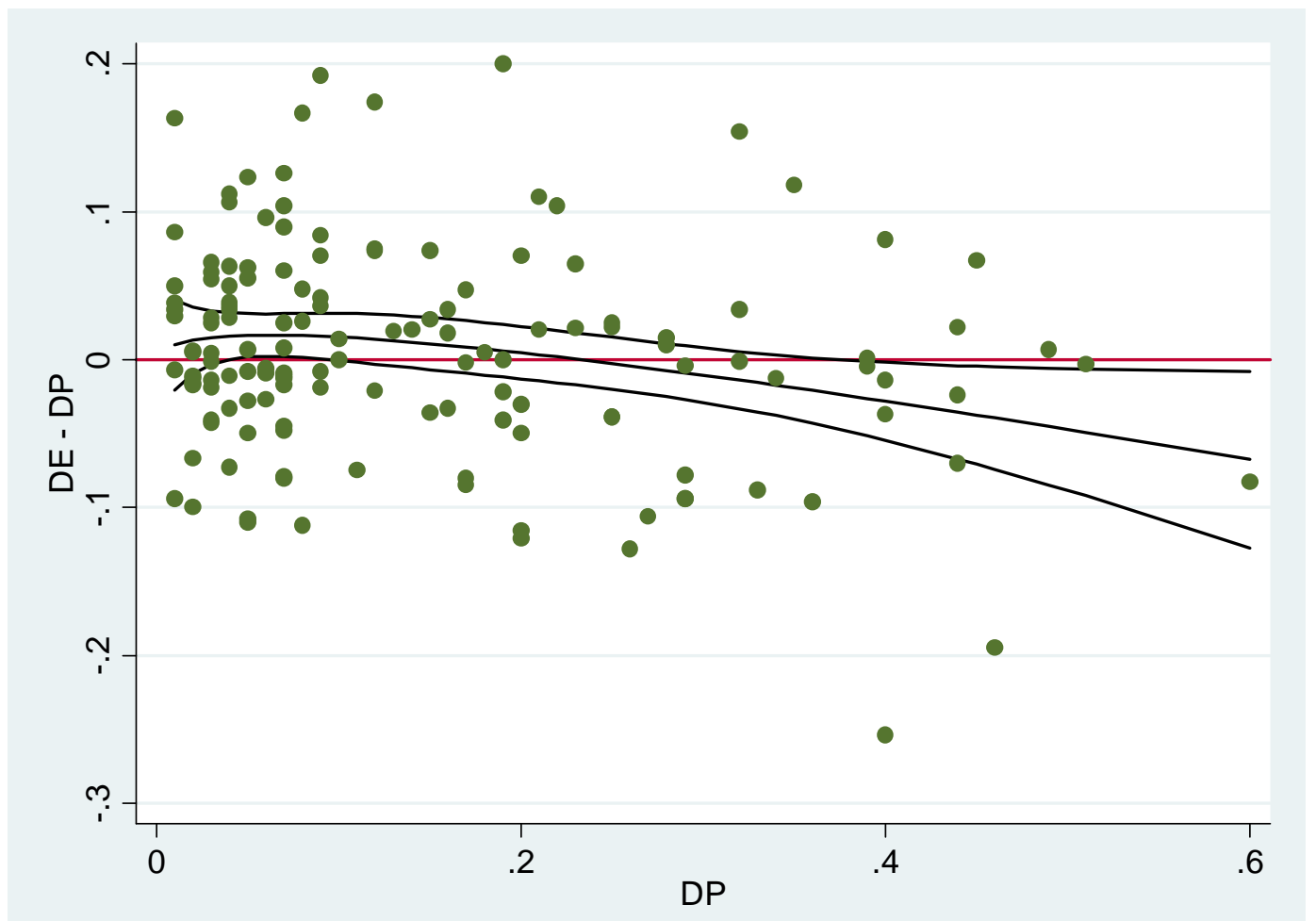


Table 1

Theoretical Predictions for the Two Different Frameworks with Benefits of Winning Equal to 10 and Costs of Voting Equal to 4.

\begin{tabular}{|c|c|c|c|c|}
\hline Distribution of Preferences & 3 vs. 4 & 2 vs. 5 & 1 vs. 6 & 0 vs. 7 \\
\hline $\begin{array}{l}\text { Probability that a supporter of the large team votes according to the } \\
\text { unique QSNE }\end{array}$ & 0.0873 & 0.0708 & 0.1399 & 0.0365 \\
\hline $\begin{array}{l}\text { Probability that a supporter of the small team votes according to the } \\
\text { unique QSNE }\end{array}$ & 0.1229 & 0.2400 & 0.8601 & \\
\hline Probability of voting according to the unique BNE & & & & \\
\hline
\end{tabular}


Table 2

Random Effect Probit Estimates of Voting Decisions in the Second Stage (Standard errors in Parentheses)

\begin{tabular}{|c|c|c|c|c|c|c|}
\hline \multirow[b]{2}{*}{ Distribution of Preferences } & \multicolumn{3}{|c|}{ The effect of majority on turnout } & \multicolumn{3}{|c|}{ The effect of beliefs on turnout } \\
\hline & 3 vs. 4 & 2 vs. 5 & 1 vs. 6 & 3 vs. 4 & 2 vs. 5 & 1 vs. 6 \\
\hline Constant & $\begin{array}{l}-0.522 \text { *** } \\
(0.170)\end{array}$ & $\begin{array}{l}-1.039 * * * \\
(0.243)\end{array}$ & $\begin{array}{l}-1.030 * * * \\
(0.407)\end{array}$ & $\begin{array}{l}-0.740 * * * \\
(0.200)\end{array}$ & $\begin{array}{l}-0.977^{* * *} \\
(0.240)\end{array}$ & $\begin{array}{l}-1.446^{* * *} \\
(0.359)\end{array}$ \\
\hline First Stage Voting Decision & $\begin{array}{c}0.207 \\
(0.143)\end{array}$ & $\begin{array}{c}0.342 * \\
(0.213)\end{array}$ & $\begin{array}{l}0.921^{* * *} \\
(0.324)\end{array}$ & $\begin{array}{c}0.232 \\
(0.145)\end{array}$ & $\begin{array}{c}0.360 * \\
(0.212)\end{array}$ & $\begin{array}{l}0.954 * * * \\
(0.335)\end{array}$ \\
\hline Round & $\begin{array}{l}-0.020 * * * \\
(0.009)\end{array}$ & $\begin{array}{l}-0.002 \\
(0.015)\end{array}$ & $\begin{array}{l}-0.002 \\
(0.022)\end{array}$ & $\begin{array}{l}-0.020 * * * \\
(0.009)\end{array}$ & $\begin{array}{l}-0.002 \\
(0.014)\end{array}$ & $\begin{array}{l}-0.004 \\
(0.023)\end{array}$ \\
\hline Majority & $\begin{array}{l}0.305^{* * *} \\
(0.108)\end{array}$ & $\begin{array}{c}0.175 \\
(0.184)\end{array}$ & $\begin{array}{l}-0.339 \\
(0.332)\end{array}$ & & & \\
\hline Pivotal Beliefs & & & & $\begin{array}{l}0.012^{* * *} \\
(0.004)\end{array}$ & $\begin{array}{c}0.002 \\
(0.004)\end{array}$ & $\begin{array}{c}0.005 \\
(0.005)\end{array}$ \\
\hline Number of Observations & 854 & 378 & 203 & 854 & 378 & 203 \\
\hline Number of Subjects & 84 & 84 & 72 & 84 & 84 & 72 \\
\hline Log Likelihood & -467.1811 & -201.2391 & -86.2301 & -468.0745 & -201.6981 & -86.2923 \\
\hline
\end{tabular}

Notes: Each column reports the estimated coefficients of a separate Random Effect Probit panel regression model in which the dependent variable is the voting decision of subjects in the second stage. $t$-statistics are in parentheses. * indicates statistically significant at $10 \%$ level, ** indicates statistically significant at $5 \%$ level; *** indicates statistically significant at $1 \%$ level. 
Table 3

Average Difference in Vote Share between Leading and Trailing Party according to Polls and Elections, 1990 - 2005 (Standard deviations in parentheses)

\begin{tabular}{|c|c|c|c|}
\hline & All Elections & $\begin{array}{l}\text { Elections Expected to be Close } \\
(\text { (DP }<0.10)\end{array}$ & $\begin{array}{l}\text { Elections Not Expected to be Close } \\
\text { (DP } \geq 0.10)\end{array}$ \\
\hline $\begin{array}{l}\text { Difference in Vote Share between Leading and Trailing Party according } \\
\text { to Elections (DE) }\end{array}$ & $\begin{array}{c}0.1530 \\
(0.1382)\end{array}$ & $\begin{array}{c}0.0645 \\
(0.0714)\end{array}$ & $\begin{array}{l}0.2592 \\
(0.1235)\end{array}$ \\
\hline $\begin{array}{l}\text { Difference in Vote Share between Leading and Trailing Party according } \\
\text { to Polls (DP) }\end{array}$ & $\begin{array}{c}0.1478 \\
(0.1344)\end{array}$ & $\begin{array}{c}0.0491 \\
(0.0258)\end{array}$ & $\begin{array}{c}0.2663 \\
(0.1147)\end{array}$ \\
\hline $\begin{array}{l}\text { Accuracy of the Polls } \\
(D E-D P)\end{array}$ & $\begin{array}{c}0.0052 \\
(0.0727)\end{array}$ & $\begin{array}{c}0.0154 \\
(0.0640)\end{array}$ & $\begin{array}{l}-0.0071 \\
(0.0807)\end{array}$ \\
\hline Number of Observations & 143 & 78 & 65 \\
\hline
\end{tabular}

Sources: Polls' data obtained from Mason-Dixon Polling and Research, Inc. 